Editorial

\title{
Formal and Methodological Approaches to Applied Linguistics (Languages Special Issue)
}

\author{
Sonja Mujcinovic * and Eduardo Gómez Garzarán * \\ Department of English, University of Valladolid, 47002 Valladolid, Spain \\ * Correspondence: sonja@fing.uva.es (S.M.); eduardo.gomez@vedruna.es (E.G.G.)
}

Received: 6 November 2019; Accepted: 13 November 2019; Published: 21 November 2019

check for updates

\section{Prologue}

This Special Issue, entitled Formal and Methodological Approaches to Applied Linguistics, brings together recently published papers on various aspects of applied linguistics presented at the AESLA37 (Spanish Society of Applied Linguistics) conference in Valladolid held on 27-29 March 2019. This issue aims to provide a better understanding of different aspects of language and to share new insights into theoretical and practical aspects in the field. It does so by addressing the study of language in its different dimensions and areas of applicability through the use of multiple methodologies and formal accounts as used by researchers in the field.

This Special Issue contains contributions from the following areas related to applied linguistics: language acquisition and language learning; language for specific purposes; language psychology, child language and psycholinguistics; sociolinguistics; discourse analysis; corpus linguistics; and lexicology and lexicography.

We are certain that the papers published in this volume offer a clear insight into the topics currently being dealt with, both from a theoretical and practical perspective, within the field of applied linguistics. Together, they provide a good example of how research on language and languages in contact are developing at present.

We would like to thank everyone who has made this Special Issue possible: authors, panel directors, and a special mention to the reviewers whose dedication has contributed considerably to this publication.

\section{Organizing Committee}

Chair: Raquel Fernández Fuertes

(English department)

Susana Álvarez Álvarez

(Spanish department)

Esther Álvarez de la Fuente

(English department)

Carmen Cuellar Lázaro

(French and German department)

Goretti Faya Ornia

(English department)

Alejandro Martínez González

(English department)

\author{
Laura Filardo Llamas \\ (English department) \\ Pedro Fuertes Olivera \\ (English department) \\ Tamara Gómez Carrero \\ (English department) \\ Eduardo Gómez Garzarán \\ (English department) \\ Lorena Hurtado Malillos \\ (French and German department) \\ Elena Jiménez García \\ (Didactics of language and literature department)
}


Nieves Mendizábal de la Cruz

(Spanish department

Susana Merino Mañueco

(Spanish department)

Leticia Moreno Pérez

(English department)

Sonja Mujcinovic

(English department)

\author{
Silvia Sánchez Calderón \\ (English department) \\ Francisco Javier Sanz Trigueros \\ (Didactics of language and literature department) \\ $\mathrm{M}^{\mathrm{a}}$ Ángeles Sastre Ruano \\ (Spanish department) \\ Radoslava Stankova Laykova \\ (English department) \\ Qianting Yuan \\ (English department)
}

\section{Coordinators of AESLA's Scientific Panels}

\author{
Ana Llinares \\ Universidad Autónoma de Madrid \\ Irina Argüelles Álvarez \\ Universidad Politécnica de Madrid Campus Sur \\ María Belén Díez BedmarUniversidad de Jaén \\ Isabel Alonso Belmonte \\ Universidad Autónoma de Madrid \\ Paloma López Zurita \\ Universidad de Cádiz \\ María del Carmen Horno Chéliz \\ Universidad de Zaragoza
}

\author{
Elena Fernández de Molina Ortés \\ Universidad de Granada \\ Francisco Alonso Almeida \\ Universidad de Las Palmas de Gran Canaria \\ Begoña Bellés FortuñoUniversitat Jaume I, Castellón \\ Moisés Almela Sánchez \\ Universidad de Murcia \\ Iraide Ibarretxe Antuñano \\ Universidad de Zaragoza \\ Miguel Ángel Candel Mora \\ Universitat Politècnica de València
}

\section{Reviewers}

María Pilar Agustín Llach, Ana Maria Anderson, Vincent Beltrán-Palanques, Jelena Bobkina, Feng Cao, Ricardo Casaño Pitach, Joseph Casillas, M. Luz Celaya, María Enriqueta Cortés de los Ríos, Do Coyle, Klaus-Dieter Rossade, María Fernández Agüero, Esther Gómez Lacabex, Diana González Pastor, Dinda L. Gorlée, Anton Granvik, Bryan Koronkiewicz, Avizia Yim Long, Silvia Molina Plaza, Natascha Müller, Jesús Manuel Nieto García, Inés Olza, Ana Isabel Otto Cantón, Vicky Papachristou, Isabel Pérez Jiménez, Paula Pérez Sobrino, M. ', Dolores Porto Requejo, Anna Rufat, Efthymia Tsaroucha, Sarah Turner, Chelo Vargas-Sierra, and several other anonymous reviewers.

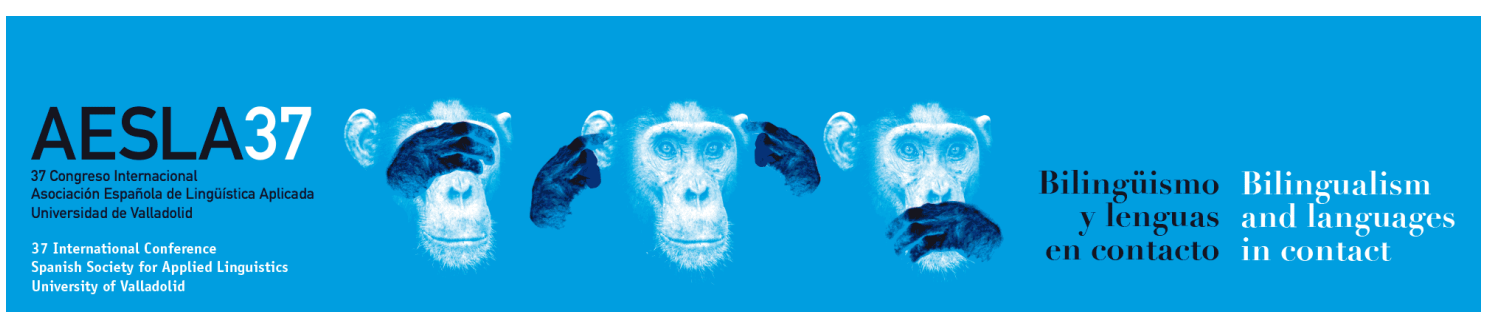

The AESLA 2019 international conference was held as part of the UVALAL (University of Valladolid Language Acquisition Lab) research activities within the framework of two research projects funded by the Castile and León Regional Government and FEDER (ref. VA009P17), and the Spanish Ministry of Science, Innovation and Universities and FEDER (ref. PGC2018-097693-B-I00). 


\section{[ $><$ ] UVALAL \\ Language \\ Acquisition \\ Laboratory \\ University of Valladolid}

\section{Junta de

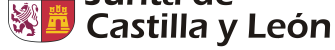

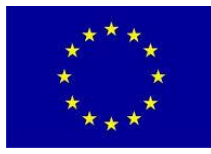

FEDER

Fondo Europeo de

Desarrollo Regional

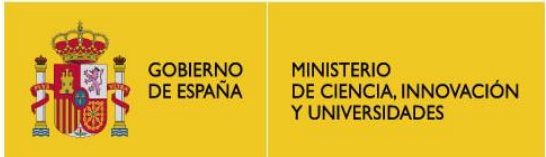

Consejería de Educación

(C) 2019 by the authors. Licensee MDPI, Basel, Switzerland. This article is an open access article distributed under the terms and conditions of the Creative Commons Attribution (CC BY) license (http://creativecommons.org/licenses/by/4.0/). 